\title{
Article \\ Genetic and Transcriptomic Analysis Reveal the Molecular Basis of Photoperiod-Regulated Flowering in Xishuangbanna Cucumber (Cucumis sativus L. var. xishuangbannesis Qi et Yuan)
}

\author{
Zhen Tian ${ }^{1}$, Molly Jahn ${ }^{2}$, Xiaodong Qin ${ }^{1}$, Hesbon Ochieng Obel ${ }^{1}$, Fan Yang ${ }^{1}$, Ji Li ${ }^{1, *}$ and Jinfeng Chen ${ }^{1}$ (i) \\ 1 State Key Laboratory of Crop Genetics and Germplasm Enhancement, College of Horticulture, \\ Nanjing Agricultural University, Nanjing 210095, China; 2015204016@njau.edu.cn (Z.T.);

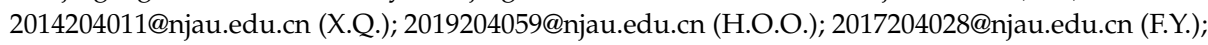 \\ jfchen@njau.edu.cn (J.C.) \\ 2 Jahn Research Group, USDA/FPL, Madison, WI 53726, USA; molly.jahn@jahnresearchgroup.net \\ * Correspondence: liji1981@njau.edu.cn; Tel.: +86-25-8439-6279
}

check for

updates

Citation: Tian, Z.; Jahn, M.; Qin, X.; Obel, H.O.; Yang, F.; Li, J.; Chen, J. Genetic and Transcriptomic Analysis Reveal the Molecular Basis of Photoperiod-Regulated Flowering in Xishuangbanna Cucumber (Cucumis sativus L. var. xishuangbannesis Qi et Yuan). Genes 2021, 12, 1064. https:// doi.org/10.3390/genes12071064

Academic Editor: Christian Chevalier

Received: 9 June 2021

Accepted: 1 July 2021

Published: 13 July 2021

Publisher's Note: MDPI stays neutral with regard to jurisdictional claims in published maps and institutional affiliations.

Copyright: (C) 2021 by the authors Licensee MDPI, Basel, Switzerland. This article is an open access article distributed under the terms and conditions of the Creative Commons Attribution (CC BY) license (https:/ / creativecommons.org/licenses/by/ $4.0 /)$.

\begin{abstract}
Xishuangbanna (XIS) cucumber (Cucumis sativus L. var. xishuangbannesis Qi et Yuan), is a botanical variety of cucumber cultivars native to southwest China that possesses excellent agronomic traits for cucumber improvement. However, breeding utilization of XIS cucumber is limited due to the current poor understanding of its photoperiod-sensitive flowering characteristics. In this study, genetic and transcriptomic analysis were conducted to reveal the molecular basis of photoperiodregulated flowering in XIS cucumber. A major-effect QTL locus DFF1.1 was identified that controls the days to first flowering (DFF) of XIS cucumbers with a span of $1.38 \mathrm{Mb}$. Whole-genome resequencing data of 9 cucumber varieties with different flowering characteristics in response to photoperiod suggested that CsaNFYA1 was the candidate gene of DFF1.1, which harbored a single non-synonymous mutation in its fifth exon. Transcriptomic analysis revealed the positive roles of auxin and ethylene in accelerating flowering under short-day (SD) light-dark cycles when compared with equal-day/night treatment. Carbohydrate storage and high expression levels of related genes were important reasons explaining early flowering of XIS cucumber under SD conditions. By combining with the RNA-Seq data, the co-expression network suggested that CsaNFYA1 integrated multiple types of genes to regulate the flowering of XIS cucumber. Our findings explain the internal regulatory mechanisms of a photoperiodic flowering pathway. These findings may guide the use of photoperiod shifts to promote flowering of photoperiod-sensitive crops.
\end{abstract}

Keywords: photoperiodic flowering process; genetic mapping; transcriptomic analysis; Xishuangbanna cucumber

\section{Introduction}

The process of flowering is one of the most remarkable phases of plant development, and it is essential for both sexual reproduction and agricultural productivity [1]. The photoperiod is one of the main important environmental signals linked to the onset and nature of flowering among the many endogenous and exogenous elements that regulate flowering and has been extensively studied in various plant species such as Arabidopsis, wheat, barley and rice [2]. Flowering is accelerated by long days and delayed by short days in Arabidopsis, a facultative long-day (LD) plant [3]. A typical short-day (SD) plant, rice, on the other hand, shows accelerated flowering when days get shorter and delayed flowering when days get longer [4]. As a general rule, reducing plant photoperiod sensitivity is important for the global cultivation of crops to ensure improved yield regardless of day length [5]. Plant breeders desire crops with the shortest possible maturity time while preserving yield in order to avoid adverse detrimental effects triggered by climate change. This further explains why photo-insensitivity is increasingly gaining more attention in plant breeding. 
The genetics and molecular biology of photoperiod-mediated flowering processes have been the subject of numerous studies. Several genes implicated in the photoperiodic flowering network have been extensively investigated. These include the florigen gene FLOWERING LOCUS T (FT) [6,7], core gene CONSTANS (CO) and Nuclear Factor Y (NFY) transcription factor complex [8,9], photoreceptors (such as PHYA, PHYB, CRY1) [10], to name a few of the most important. Previous study has treated time to flower as the target trait used to evaluate photoperiod sensitivity [11]. The flowering process consists of multiple different and important stages at which these effects might be exerted. Under the appropriate induction conditions (photoperiod, temperature and hormones, etc.), the shoot apical meristem gradually changes to the inflorescence meristem, then the meristem forms flower meristems and gradually differentiates into various flower organs, finally forming flowers [12]. Many studies have focused on the effects of photoperiod on flowering processes; however, it is still not clear which stage(s) of flowering are affected by photoperiod. Furthermore, it is necessary to examine significant changes that occurs in the most prominent points.

Cucumber, Cucumis sativus L. $(2 \mathrm{n}=2 \mathrm{x}=14)$, is an economically significant species globally, with the fruit being consumed as a vegetable in variety of cuisines all around the world. The genetically homogeneous, inbred breeding line used in this study is called Xishuangbanna (XIS) cucumber (Cucumis sativus L. var. xishuangbannesis Qi et Yuan [13]) and is a typical SD plant. A wide range of various types of cucumber species have spread across China. Their photoperiod sensitivity varies with latitude. In China, three types of cucumbers have been developed based on photoperiod sensitivity: North China type, South China type, and Southwest China type (mainly XIS cucumber) [14]. The proposed order of photoperiod sensitivity is Southwest China type $>$ South China type $>$ North China type [15]. Photoperiod has no or little influence on the flowering of day-neutral North China type; however, the short-day XIS cucumber is sensitive to photoperiod changes [16]. The SD flowering character of XIS cucumber limits gene flow with other ecotypes due to asynchronous flowering. Additionally, the delay in flowering time extends the time the crop is in the field, thereby increasing risk of exposure to adverse weather before harvest that may negatively affecting crop yield. This study was undertaken to examine the photoperiodic regulation mechanisms that determine XIS cucumber flowering.

In this study, we applied SSR-based mapping and QTL-Seq analysis on the days to first flowering. RNA sequencing (RNA-Seq) analysis was conducted in the floral primordia initiation stage and the floral organ development stage to investigate the differential expression of specific genes. Finally, combined with the RNA-Seq data, we constructed the co-expression network of candidate genes that contribute to genetic variation in the photoperiod-mediated flowering pathway. Our study advances our understanding of the regulation mechanisms of photoperiodic flowering process.

\section{Materials and Methods}

\subsection{Plant Materials and SSR-Based Mapping Analysis}

CC3 (maternal line, P1) is a typical North China market type, which is insensitive to photoperiod. SWCC8 (paternal line, P2) is derived from the XIS cucumber, which is sensitive to photoperiod and requires a SD treatment for flowering in temperate regions. A population of 124 recombinant inbred lines (RILs, $\mathrm{F}_{9}$ ) from the cross of CC3 and SWCC8 [17] was used for mapping the target trait days to first flowering (DFF). The data from the RIL9 population were recorded in spring, 2016 (for each individual of the RIL, five plants were screened) and fall, 2016 (for each individual of the RIL, six plants were screened) in the greenhouse at Nanjing Agricultural University. DFF refers to the days from sowing to the first flower blooming (either male or female flower). Combining the previous linkage map [17] and phenotypic data of the RIL9 population, the QTL mapping on DFF was performed using the composite interval mapping (CIM) function of WinQTL Cartographer Version $2.5[18,19]$. The QTLs were named according to their chromosome location, trait 
name and the season. Sdff1.1 and Fdff1.1 represent the QTLs for DFF on chromosomes 1 in spring and fall detected by QTL mapping.

\subsection{QTL-Seq Analysis}

An $\mathrm{F}_{2}$ population from the $\mathrm{CC} 3 \times$ SWCC 8 of 234 individuals was also used to further confirm the trait DFF by QTL-Seq analysis. All $\mathrm{F}_{2}$ plants were categorized according to the DFF. Two bulked DNA pools (early flowering pool, E-pool, 36-40 d; late flowering pool, L-pool, $>52 \mathrm{~d}$ ) were generated by mixing an equal amount of DNAs from $17 \mathrm{~F}_{2}$ plants in the 2017 fall using the CTAB method [20]. Thus, four Illumina libraries, two extreme bulks and parental lines CC3 and SWCC8, were sequenced individually on an Illumina HiSeq 2500 (Illumina lnc., San Diego, CA, USA). All sequencing in this study was carried out by Novogene. The raw data of QTL-Seq was uploaded successfully to NCBI Short Read Archive (SRA) under accession number SRP150560. All clean reads were aligned to the reference genome (9930 Version 2) [21]. SNP-calling was performed by SAM tools. SNP-index was assigned with the following principles: the same as CC3, SNP-index was 0; completely different, SNP-index was 1 . Then the $\Delta$ (SNP-index) was calculated by subtraction the SNP-index of E-pool from that of L-pool. The average of SNP-index was calculated to reflect its distribution in a $1 \mathrm{Mb}$ window size and $1 \mathrm{~kb}$ increment. The $\Delta$ (SNP-index) value were used to identify the candidate QTLs on DFF, 1000 permutation tests and 95\% confidence level. The QTLs were named according to its trait name and chromosome location. For example, dff1.1 refers to the QTL for DFF on chromosome 1 using QTL-Seq.

\subsection{Prediction and Verification of Candidate Genes Regulating DFF}

The QTL-Seq results and re-sequencing data of nine cucumber materials was performed to explore the possible candidate genes with nucleotide variations, located in the QTLs. Among them, CC3, CCMC and 9930 are typical North China type cucumber which are insensitive to photoperiod; Cuiyu8, L8 and Erzaozi belong to the South China type cucumber, showing a photoperiod response between the North and Southwest China type; the photoperiod-sensitive XIS cucumbers SWCC8, SWCC20 and SWCC23 were also used in this study. The re-sequencing raw data was uploaded to NCBI with the trace number SRP218666. The expression patterns of candidate genes were identified in multiple organs and different photoperiod regimes. Combined with the RNA-Seq data, the predicted co-expression network of candidate genes was presented.

\subsection{Plant Materials and Growth Conditions of RNA-Seq Analysis}

Photoperiod-sensitive XIS cucumber, SWCC8, was used for the RNA-Seq analysis [17]. Plants perceive photoperiod changes through leaves [6], therefore the transcriptome of leaves (the second true leaf below the apex) was analyzed by RNA-Seq after SD and equalday (ED) treatments. Seeds of SWCC8 were sown into a medium (peat:vermiculite $=3: 1$ ) and grown in a chamber at $12 \mathrm{~h} / 12 \mathrm{~h}$ (light/dark), $28^{\circ} \mathrm{C} / 18{ }^{\circ} \mathrm{C}$ (day/night), $80 \%$ relative humidity, and $800 \mu \mathrm{mol} \cdot \mathrm{m}^{-2} \cdot \mathrm{s}^{-1}$ photo flux density. Two weeks after germination, uniformly-sized seedlings with one fully unfolded true leaf were moved to two different chambers, each with a different photoperiod treatment: SD $(8 \mathrm{~h} / 16 \mathrm{~h}$, day/night) and ED (12 h/12 h, day/night), whereas all other parameters were the same.

Leaf samples were collected from the XIS cucumber SWCC8 at varying times under SD and ED conditions (Table 1). Control samples taken at 0 days after photoperiod treatment (DAT), that is, before photoperiod treatments, were defined as initial period (IP). Based on observations of the shoot apical meristem (SAM) and later flower bud development using light microscopy of paraffin sections, samples harvested on 7 DAT, 14 DAT and 21 DAT were treated as the floral primordia initiation stage, of which the RNA was mixed to define SD1 and ED1 samples respectively. Samples collected at 37 DAT and 44 DAT were likewise pooled and considered as the floral organ development stage under SD treatment. Due to the delayed flowering, the floral organ development stage under ED treatment included as 
ED2 (37 DAT and 44 DAT were pooled) and ED3 (52 DAT and 59 DAT were pooled). Each sample consisted of three biological replicates collected from three individual plants. The materials were immediately placed in liquid nitrogen after harvest and stored at $-80^{\circ} \mathrm{C}$.

Table 1. The detailed experimental plan of RNA-Seq.

\begin{tabular}{|c|c|c|c|}
\hline $\begin{array}{l}\text { Photoperiod } \\
\text { Regimes }\end{array}$ & Initial Period & $\begin{array}{l}\text { Floral Primordial } \\
\text { Initiation Stage }\end{array}$ & Floral Organ Development Stage \\
\hline $\mathrm{SD}(8 \mathrm{~h} / 16 \mathrm{~h})$ & \multirow{2}{*}{ IP (0 DAT) } & $\operatorname{SD} 1(7,14,21 \mathrm{DAT})$ & SD2 $(37,44$ DAT $)$ \\
\hline $\operatorname{ED}(12 \mathrm{~h} / 12 \mathrm{~h})$ & & $\operatorname{ED1}(7,14,21 \mathrm{DAT})$ & ED2 (37, 44 DAT) \\
\hline
\end{tabular}

DAT, days after photoperiod treatment.

According to the sampling time of RNA-Seq, the SAM (0 DAT, 7 DAT, 14 DAT and 21 DAT) and later flower buds (37 DAT, 44 DAT, 52 DAT and 59 DAT) were harvested, and were fixed in formalin-acetic acid-alcohol (FAA; methanol:acetic acid:50\% ethyl alcohol = 5:5:90) and were then embedded, sectioned, dewaxed, and hematoxylin and eosin (HE)-staining [22]. All of the specimens were photographed under a BX41 microscope (Olympus, Tokyo, Japan).

\subsection{RNA Extraction, Library Preparation and Sequencing}

Total RNA was extracted using the Trizol Reagent (Invitrogen). Degradation and contamination of RNA were assessed on 1.5\% agarose gels. RNA integrity was checked using the Nano 6000 Assay Kit of the Agilent Bioanalyzer 2100 system (Agilent Technologies, Palo Alto, California, CA, USA). High-quality RNA was pooled in equal quality following the description in the preparation of RNA-Seq materials, and $5 \mu \mathrm{g}$ bulked RNA was used for later cDNA library preparations. Sequencing library construction was performed using the NEBNext ${ }^{\circledR}$ Ultra $^{\text {TM }}$ RNA Library Prep Kit for Illumina ${ }^{\circledR}$ (NEB, Ipswich, Massachusetts, MA, USA) following the manufacturer's protocol. The prepared libraries were sequenced on an Illumina Hiseq 2500 platform, and $150 \mathrm{bp}$ paired-end reads were generated. The RNA-Seq data was deposited in NCBI under accession number SRP151164.

\subsection{RNA-Seq Data and Enrichment Analysis}

Clean reads were obtained by removing low-quality reads, adapter, and reads containing ploy-N from raw data. Then, they were mapped to the cucumber genome (http:/ / cucurbitgenomics.org/organism/2 accessed on 1 July 2021, ver. 2i) [21] using the software TopHat (version 2.0.12) [23]. Expression levels of each gene were measured as reads per kilobase of coding sequence per million mapped reads (RPKM) [24]. DESeq $R$ package (version 1.18.0) [25] was used to analysis differential expressed genes (DEGs) of two samples, with false discovery rate (FDR) $\leq 0.001$ and $\left|\log _{2} \mathrm{FC}\right| \geq 1$. KEGG enrichment analysis was performed using the online website (https:/ /www.omicshare.com/ accessed on 1 July 2021) with default instructions, and a $p$-value $<0.05$ was used to determine significantly enriched KEGG entries.

\subsection{Quantitative Real-Time PCR Analysis of RNA-Seq Data}

Quantitative real-time PCR (qRT-PCR) analysis was carried out with samples described following those in RNA-Seq. Primer Premier 5.0 was used to design suitable primers (Table S1), and primers were synthesized by TSINGKE Biotech. cDNAs were reverse-transcribed using the PrimeScript ${ }^{\mathrm{TM}}$ RT reagent Kit with gDNA Eraser (Takara, Dalian, China), and qRT-PCR analysis was performed on a iCycler Real-Time PCR Detection System (Bio-Rad, Berkeley, California, CA, USA) using TaKaRa SYBR Premix Ex $\mathrm{Taq}^{\mathrm{TM}}$ (Tli RNaseH Plus, Takara, Dalian, China) with three replications. The cucumber $\beta$-actin gene (ID number: Csa2G301530) was used as the internal control to normalize the expression data. The relative expression of each gene was calculated using the $2^{-\Delta \Delta C T}$ method [26]. 


\subsection{Assay of Phenotypic and Physiological Parameters}

Phenotypic data from SWCC8 plants were collected when the first flower opened under SD $(8 \mathrm{~h} / 16 \mathrm{~h})$ and ED $(12 \mathrm{~h} / 12 \mathrm{~h})$ treatments. The corresponding data of the days to flower, nodes and plant height when the first flower open was recorded. The assays of physiological variables were carried out by the RNA-Seq materials. Coomassie Brilliant Blue G-250 method was used for soluble protein assay [27]. The concentration of phytohormones, auxin and ethylene, were analyzed by the ELISA Kits (Sbjbio Life Science, Nanjing, China) with three biological replicates. The content of starch and sucrose were determined by the 3,5-dinitrosalicylic acid spectrophotometry [28].

\subsection{Weighted Gene Co-Expression Network Analysis}

Weighted gene co-expression network analysis (WGCNA) was performed by the RNA-Seq data (WGCNA package in R3.6.1). And genes with an averaged RPKM $\geq 5$ were retained (14,492 genes) for the WGCNA analysis. The modules were obtained using the editable R program, followed with key parameters designed as: the soft threshold power was 16, and TOMType was signed. Then the gene dendrogram was used for modules identification (minModuleSize was 30). The similar modules were merged to form the final modules, with 0.25 mergeCutHeight. The module eigengene value was calculated and used to test the correlation between modules and samples. According to the topological overlap measure in each module, the associated gene pairs with corresponding edge weight value was obtained. Connectivity was defined as the sum of the edges of a node. The co-expression network was visualized using Cytoscape_v.3.7.2.

\section{Results}

\subsection{Both Vegetative and Reproductive Growth Were Regulated by Photoperiod in XIS Cucumber}

The short-day XIS cucumber SWCC8 is sensitive to photoperiod. Bo et al. [16] showed that an $8 \mathrm{~h}$ day $/ 16 \mathrm{~h}$ night regime is the best photoperiod regime to induce early SWCC 8 flowering. In our study, we designed two different light cycles, $8 \mathrm{~h} / 16 \mathrm{~h}$ (SD) and $12 \mathrm{~h} / 12 \mathrm{~h}$ (ED) day/night, to define the photoperiod responses of XIS cucumber SWCC8. The influence of photoperiod on flowering is exhibited as days to flower, number of nodes, plant height (Table 2). Because male flowers are first to bloom under both light cycles, we compared times until the appearance of the first male flower. With increasing lengths of daylight, the days to first flowering increased, reaching the level of significant difference. The flowering time under $12 \mathrm{~h} / 12 \mathrm{~h}(98 \mathrm{~d}$ ) was 18 days longer than that under $8 \mathrm{~h} / 16 \mathrm{~h}$ $(80 \mathrm{~d})$. The nodes and plant height of first flowering also showed a similar tendency and significant difference under two light cycles. Compared with the SD regime, under ED treatment SWCC8 delays its flowering process and has more vigorous vegetative growth, accompanied by more nodes and increased plant height.

Table 2. The detailed information of XIS cucumber, SWCC8, when the first flower opens under two photoperiod regimes.

\begin{tabular}{cccc}
\hline Treatments (Day/Night) & DFF (d) & NFF & ph (cm) \\
\hline SD $(8 \mathrm{~h} / 16 \mathrm{~h})$ & $80.0 \pm 0.0 \mathrm{~b}$ & $11.3 \pm 2.3 \mathrm{~b}$ & $55.5 \pm 2.5 \mathrm{~b}$ \\
ED $(12 \mathrm{~h} / 12 \mathrm{~h})$ & $98.0 \pm 0.0 \mathrm{a}$ & $16.0 \pm 0.0 \mathrm{a}$ & $68.2 \pm 2.4 \mathrm{a}$ \\
\hline
\end{tabular}

The male flowers often to bloomed first under two light cycles, thus the male flower was used to indicate the days to first flowering in this study. The significant difference analysis was distinguished by $\mathrm{a}, \mathrm{b}(p<0.05)$. DFF, the days to first flowering; NFF, the nodes to first flowering; ph, the plant height to first flowering.

In order to discover the effect of the photoperiod on the internal structures, paraffin sections were prepared to evaluate morphological differences in meristematic tissues throughout the flowering process. Before photoperiod treatments, during the initial period of sampling, the SAM was simple and tiny, bearing only inflorescence meristem (IM) and leaf primordia (LP) (Figure 1a). Later, the floral primordia (FP) appeared at similar times under both light cycle regimes (Figure 1c,f). The final floral organ development, however, 
was delayed under ED relative to SD condition. The flower buds underwent differentiation into sepals, petals, stamens, and carpels in the SD2 (Figure 1h,i), compared with only sepals and petals at ED2 (Figure 1j,k). The flower buds were differentiated into sepals, petals, stamens (Figure 1l), and later carpels (Figure 1m) until the ED3 stage under ED regime. The SD treatment accelerating the flowering process compared with ED in XIS cucumber SWCC8. In summary, photoperiod also affects the reproductive growth of XIS cucumber, and the effects of photoperiod are mainly evident at the stage of floral development where floral organs differentiate.

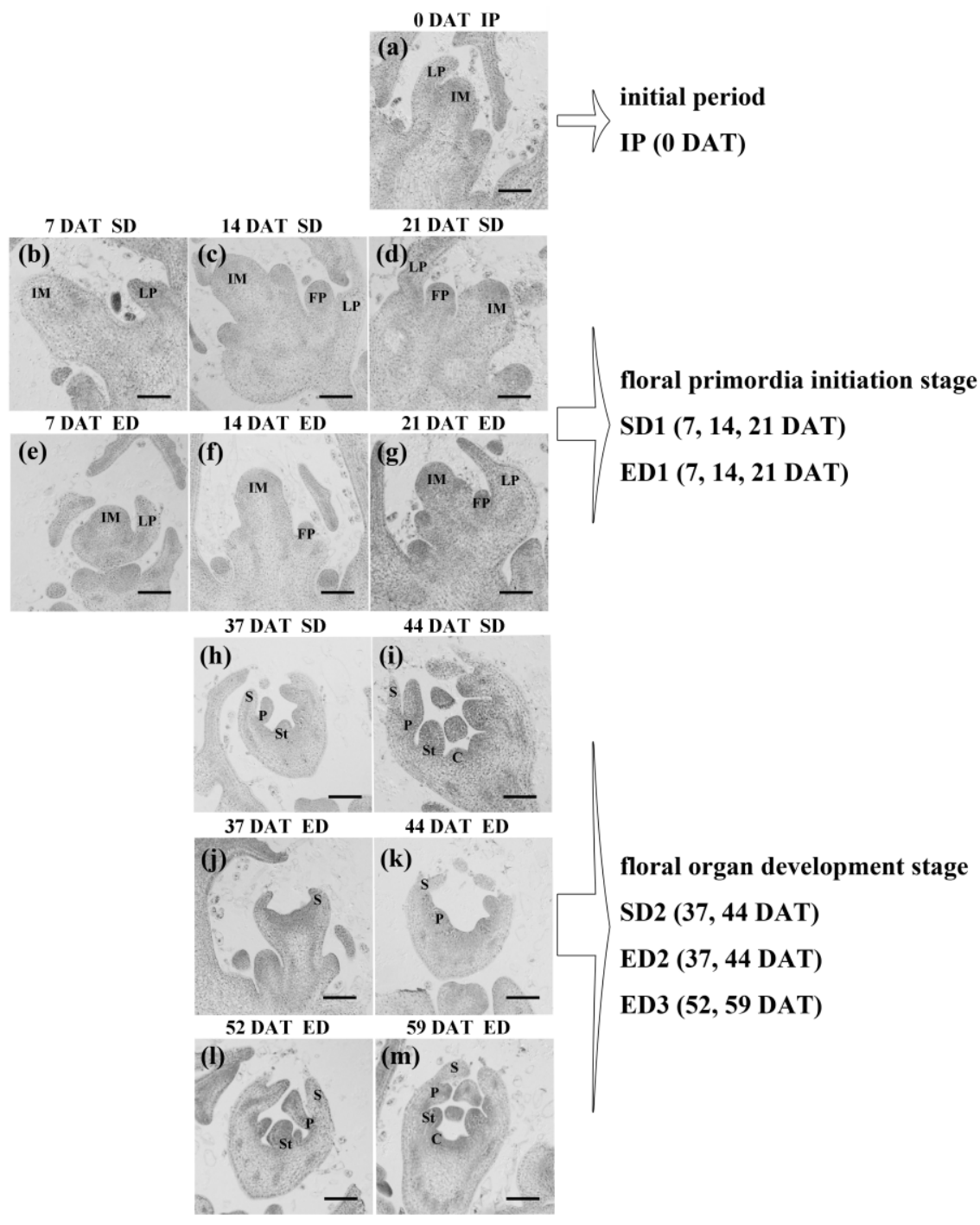

Figure 1. Before the first flower opens, the morphological characterizations of shoot apical meristem (SAM, 0 DAT, 7 DAT, 14 DAT, 21 DAT) and later flower bud (37 DAT, 44 DAT, 52 DAT and 59 DAT) corresponding to the time points of RNA-Seq. (a) The SAM before photoperiod treatments, namely initial period (IP). (b-d,h,i) The SAM and flower buds under short-day (SD, $8 \mathrm{~h} / 16 \mathrm{~h}$ ) light cycle. $(\mathbf{e}-\mathbf{g}, \mathbf{j}-\mathbf{m})$ The SAM and flower bud under equal-day (ED, $12 \mathrm{~h} / 12 \mathrm{~h}$ ) condition. DAT, days after photoperiod treatment; IM, inflorescence meristem; FP, floral primordia; LP, leaf primordia; S, sepal; P, petal; St, stamen; C, carpel. Bars $=100 \mu \mathrm{m}$.

\subsection{Stable QTL Loci Were Obtained on DFF by the SSR-Based Mapping}

Plant photoperiod-mediated flowering is related to multiple traits. Previous studies showed that flowering time is often used as an indicator to measure plants' photoperiod response $[11,29]$. In this study, North China type cucumber CC3, not sensitive to photoperiod, and Southwest China type cucumber SWCC8, sensitive to photoperiod, were used 
as parental lines to construct populations to predict the possible candidate loci and genes related to the target trait DFF.

In the two seasons' field experiments, the female parent $\mathrm{CC} 3$ always flowered earlier than most RIL lines and male parent SWCC8; and the flowering time of $\mathrm{F}_{1}$ plants is close to CC3 (Figure S1a,b), suggested that the early flowering allele in CC3 might be dominant. The phenotypic statistics of DFF in the RIL9 population showed a continuous distribution in 2016 spring and fall, a standard normal distribution in spring (kurtosis, 0.28; skewness, 0.32 ) and a bimodal distribution in fall (kurtosis, -1.19 ; skewness, 0.01) (Table 3). The previous SSR-based genetic map [17], which contains 269 SSR markers with a total length of $705.9 \mathrm{cM}$, was employed to map the target trait DFF after combining the phenotypic data. Detailed results including detected QTLs, chromosomes, peak location, marker interval, LOD score, total phenotypic variance $\left(R^{2}\right)$, and additive effect were listed in Table 4. Only two QTLs of DFF, Sdff1.1 and Fdff1.1, were identified by the SSR-based mapping; both of their phenotypic effects on variation on DFF were $>10 \%\left(R^{2}=10.6 \%\right.$ and $21.7 \%$, respectively), establishing these two loci as major-effect QTLs on the trait, DFF. The negative additive effect of Sdff1.1 and Fdff1.1 suggested that allele that comes from SWCC8 could delay the flowering time.

Table 3. Phenotypic variation of DFF in the RIL9 population at 2016 spring and fall.

\begin{tabular}{ccccccc}
\hline \multirow{2}{*}{ Seasons } & \multicolumn{2}{c}{ Parents } & \multicolumn{3}{c}{ RIL Populations } \\
\cline { 2 - 7 } & CC3 & SWCC8 & Range & Mean \pm SD & Kurtosis & Skewness \\
\hline 2016 spring & $47.2 \pm 0.9$ & $87.8 \pm 0.6$ & $45.0 \sim 90.3$ & $62.8 \pm 0.7$ & 0.28 & 0.32 \\
2016 fall & $32.5 \pm 0.6$ & $69.5 \pm 0.9$ & $32.0 \sim 55.0$ & $42.5 \pm 0.5$ & -1.19 & 0.01 \\
\hline
\end{tabular}

Table 4. QTLs detected on DFF by the SSR-based mapping.

\begin{tabular}{ccccccc}
\hline QTL Loci & Chr. & $\begin{array}{c}\text { LOD } \\
\text { Score }\end{array}$ & $\mathbf{R}^{\mathbf{2}} \mathbf{( \% )}$ & $\begin{array}{c}\text { Additive } \\
\text { Effects }\end{array}$ & $\begin{array}{c}\text { Peak Location } \\
\mathbf{( c M )}\end{array}$ & Marker Interval \\
\hline Sdff1.1 & 1 & 3.5 & 10.6 & -7.9 & 86.2 & SSR16841-SSR23049 \\
Fdff1.1 & 1 & 4.9 & 21.7 & -3.6 & 88.8 & SSR05723-SSR22638 \\
\hline
\end{tabular}

\subsection{DFF1.1 Is a Major Effect QTL Controlling Photoperiod Responsive Flowering}

The QTL loci in the SSR-based QTL mapping were then confirmed using the QTL-Seq analysis on DFF. The DFF showed a continuous distribution (Figure S1c) in the $\mathrm{F}_{2}$ population derived from CC $3 \times$ SWCC 8 (kurtosis, -0.36 ; skewness, 0.58 ), which meets the characteristics of quantitative trait. The Illumina high-throughput sequencing resulted in $11,084,076,600$ bp and 13,579,662,300 bp clean reads for the L-pool and E-pool, respectively (Table S2). The mapping rate of the four Illumina libraries was $83.59-85.45 \%$. The comparison results were normal and can be used for subsequent variation detection analysis. By combining the information of SNP-index in E-pool and L-pool, $\Delta$ (SNP-index) was calculated and plotted against the genome positions. Finally, we identified four QTLs on DFF with 95\% confidence level, namely dff1.1, dff3.1, dff6.1 and dff7.1 (Figure 2a).

Previous studies showed that the mapping results on flowering time were located at the similar intervals on chromosomes 1 and 6 (Table 5), when the parent lines contain cucumber varieties that are sensitive to photoperiod [17,30,31]. In this study, combined QTL mapping (Sdff1.1 and Fdff1.1) and QTL-Seq (dff1.1, dff3.1, dff6.1 and dff7.1) results together, three QTLs were located on chromosome 1, and all were located in a similar region with the overlapping area (DFF1.1) spanned 1.38 Mb (21.65-23.03 Mb) (Figure 2b). By combining with the previous studies, we suggested that there is a conservative QTL DFF1.1 of the photoperiodic flowering time on chromosome 1. 


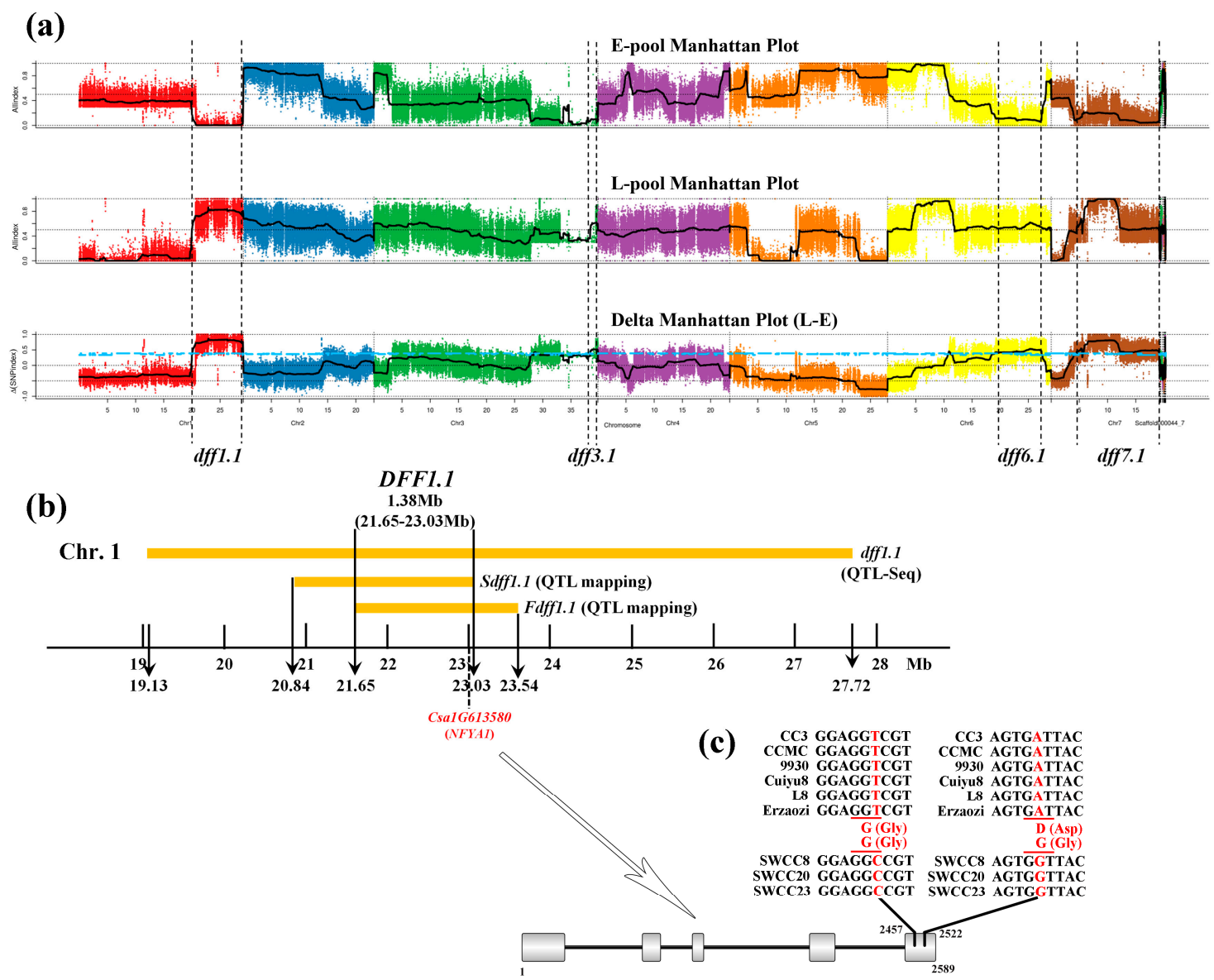

Figure 2. Summary of the mapping results on DFF. (a) The QTL-Seq results by the $\mathrm{F}_{2}$ population derived from the cross of CC3 and SWCC8. (b) The overlapping region of DFF on chromosome 1. (c) The structure variation of candidate gene CsaNFYA1 (Csa1G613580). The only one non-synonymous mutation was detected at 2522 (D to G, Aspartate to Glycine).

Table 5. Summary of QTLs detected on the days to first flowering.

\begin{tabular}{|c|c|c|c|c|c|}
\hline Number & QTL Loci & Chr. & \multicolumn{2}{|c|}{ Parental Lines (Left, Female Parent; Right, Male Parent) } & References \\
\hline 1 & dff1.1 & 1 & & \multirow{5}{*}{$\begin{array}{l}\text { SWCC8 (semi-wild XIS cucumber, } \\
\text { sensitive) }\end{array}$} & \multirow{4}{*}{$\begin{array}{l}\text { QTL-Seq results in this study } \\
\text { (from } 1 \text { to } 4 \text { ) }\end{array}$} \\
\hline 2 & dff3.1 & 3 & \multirow{5}{*}{$\begin{array}{l}\text { CC3 (cultivated cucumber, } \\
\text { insensitive) }\end{array}$} & & \\
\hline 3 & $d f f 6.1$ & 6 & & & \\
\hline 4 & dff7.1 & 7 & & & \\
\hline 5 & Sdff1.1 & 1 & & & SSR-based mapping results in this \\
\hline 6 & Fdff1.1 & 1 & & \multirow{6}{*}{ WI7221 (wild cucumber, sensitive) } & study (from 5 to 6 ) \\
\hline 7 & $f t 1.1$ & 1 & \multirow{5}{*}{$\begin{array}{l}\text { Gy14 (cultivated cucumber, } \\
\text { insensitive) }\end{array}$} & & \multirow{5}{*}{ Sheng et al. [31] (from 7 to 10 ) } \\
\hline 8 & $f t 1.1$ & 1 & & & \\
\hline 9 & $f t 6.3$ & 6 & & & \\
\hline 10 & $f t 6.3$ & 6 & & & \\
\hline 11 & $f f t 1.1$ & 1 & & & \\
\hline 12 & mft1.1 & 1 & \multirow{4}{*}{$\begin{array}{l}\text { WI7176 (semi-wild XIS } \\
\text { cucumber, sensitive) }\end{array}$} & \multirow{4}{*}{$\begin{array}{l}\text { WI7200 (landrace originally } \\
\text { collected from Thailand, insensitive) }\end{array}$} & \multirow{4}{*}{ Pan et al. [30] (from 11 to 15) } \\
\hline 13 & fft5.1 & 5 & & & \\
\hline 14 & $f f t 6.2$ & 6 & & & \\
\hline 15 & mft6.2 & 6 & & & \\
\hline 16 & $f f t 1.1$ & 1 & \multirow{2}{*}{$\begin{array}{c}\text { CC3 (cultivated cucumber, } \\
\text { insensitive) }\end{array}$} & \multirow{2}{*}{$\begin{array}{l}\text { SWCC8 (semi-wild XIS cucumber, } \\
\text { sensitive) }\end{array}$} & \multirow{2}{*}{ Bo et al. [17] (from 16 to 17) } \\
\hline 17 & $f f t 6.1$ & 6 & & & \\
\hline
\end{tabular}

To identify the candidate genes for the QTL, DFF1.1, we took the mapping results and re-sequencing data into consideration. Initially, QTL-Seq detected the large interval dff1.1 (19.13 Mb-27.72 Mb) on chromosome 1. After selecting the most significant polymorphic SNP and Indel sites in the two bulked pools, a total of 298 nonsynonymous mutation sites in 150 nonsynonymous mutation genes $(\Delta($ SNP-index $)>0.7)$ were identified (Table S3), 
but only 15 nonsynonymous mutation genes were left in the $1.38 \mathrm{Mb}$ overlapping region DFF1.1 (Table S4). The re-sequencing data of cucumber varieties with different photoperiod sensitivity was used for the prediction of candidate genes (Table S5). Finally, four genes, Csa1G605740, Csa1G611280, Csa1G613550 and Csa1G613580 (marked in red in Table S4) followed the pattern: North (CC3, CCMC) and South (Cuiyu8, L8, Erzaozi) China type cucumbers were same to the reference genome 9930, whereas all of the Southwest XIS cucumbers (SWCC8, SWCC20, SWCC23) were consistent with each other and different from the reference genome 9930. After taking the annotation information into consideration, gene Csa1G613580 (hereafter CsaNFYA1), which can recognize the CCAAT-box cis-acting element in the promoter region of the $F T$ gene in the photoperiodic flowering pathway [32,33], was left as a candidate for DFF1.1. The alignment of nucleotide sequences showed that two SNPs were detected in the exon region of CsaNFYA1 compared with the reference genome 9930, located in the fifth exon at nucleotides 2457 ( $T$ to C) and 2522 (A to G); however, only one non-synonymous mutation was detected (D to G, Aspartate to Glycine), which came from the mutation of 2522 (A to G) (Figure 2c).

\subsection{The Response of Hormone Was Significantly Different under SD and ED Regimes}

A time-course RNA-seq analysis of leaves was performed under two kinds of photoperiod treatments. A total of 18 libraries and 56.62 (SD1-3)-60.92 (ED3-2) million raw reads were generated. After trimming and filtering, the clean reads were mapped to the cucumber genome, and the number of perfectly matched reads ranged from 22.09 (SD2-3, $40.09 \%$ ) to 27.16 (IP-2, 46.84\%) million (Table S6). The qRT-PCR results of six DEGs showed similar expression patterns compared with the RNA-Seq data (Figure S2), and all of the Pearson's correlation coefficients were $>0.8$, further confirming the reliability of the RNA sequencing results. The correlation clustering and principal component analysis (PCA) of the 18 RNA-Seq datasets were performed (Figure S3). A close correlation between biological replicates was obtained. PCA analysis showed three distinct groups corresponding to the initial period, the floral primordia initiation stage (SD1 and ED1), and the floral organ development stage (SD2, ED2 and ED3). Even though ED2 was an extended phase under ED treatment, PCA showed that it had a very similar expression pattern to SD2 and ED3, which means that under the ED treatment, gene expression progressed to the floral organ development stage.

To reveal the causes of the apparent promotion of flowering process under SD when compared with the ED treatment, the significant DEGs $\left(\left|\log _{2} \mathrm{FC}\right| \geq 1\right)$ were further analyzed in the comparisons between SD and ED photoperiod regimes, i.e., SD1/ED1, SD2/ED2 (the comparisons at the same time) and SD2/ED3 (the comparisons at the similar stage). A total of 2968 significant DEGs were produced in the three comparisons. And more DEGs were gathered in SD1/ED1 (1666) compared with SD2/ED2 (700) and SD2/ED3 (1273) (Figure 3a). Transcription factors (TFs) regulate plant growth and development through various signal transduction pathways [34]. Combined with previous studies, multiple flowering-related TFs were detected in the DEGs, such as bHLH, MADS-box, MYB and WRKY [35-37]. Noteworthy, ERF and NAC TFs were marked in top three in the three comparisons (Figure $3 b$ ), which used to be involved into the flowering process especially the regulation of floral organ development [38,39].

The KEGG enrichment analysis (Figure 3a) showed that entries related to replication and repair, signal transduction and material metabolism were detected in the comparison of SD1/ED1. Except signal transduction and metabolism terms, SD2/ED2 and SD2/ED3 comparisons were marked by translation and environmental adaptation entries. Notably, the "plant hormone signal transduction" entry was enriched in all three kinds of comparisons. Among them, DEGs involved in ethylene and auxin signal transduction hold a relatively high and stable proportion in the three comparisons (Figure 3c), in agreement with the pivotal roles of ethylene and auxin in the initiation of flowering and flower development [40-42]. The concentration of ethylene and auxin showed an upward trend under both light cycles, and their concentration under SD was almost higher than that 
under ED condition, especially at the floral organ development stage (Figure 3d). The higher concentration of ethylene and auxin under SD regime was consistent with the early flowering under $\mathrm{SD}$, further implied the positive effects of ethylene and auxin in promoting flowering in XIS cucumber.

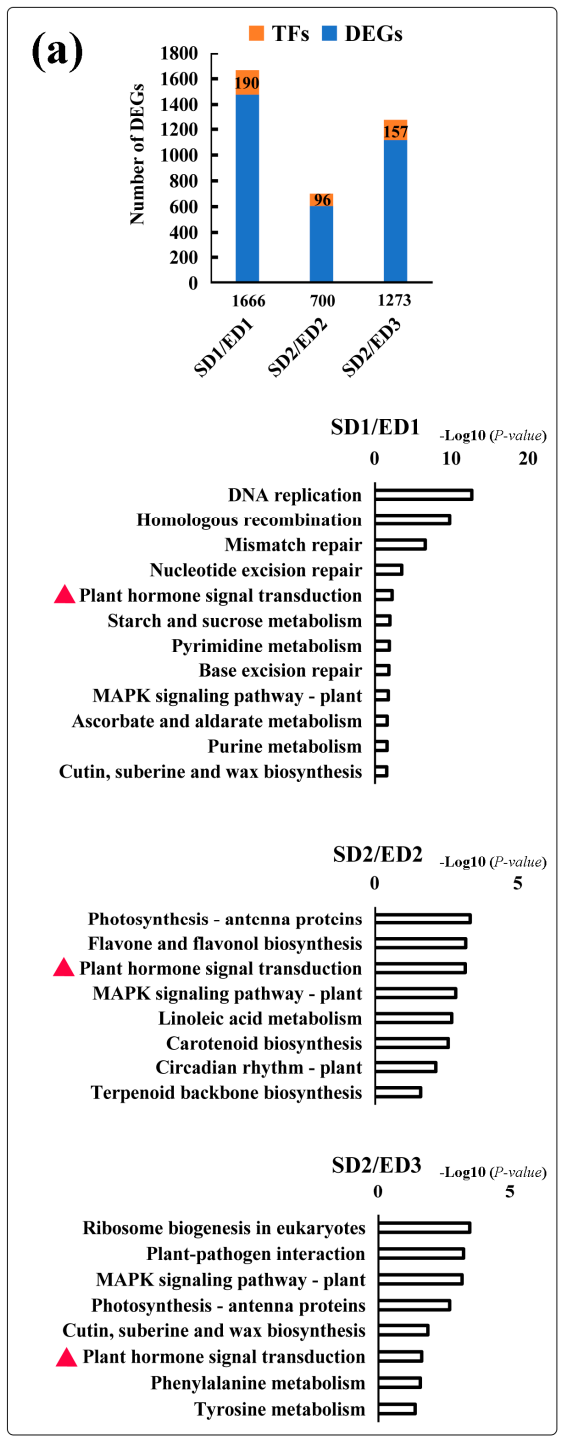

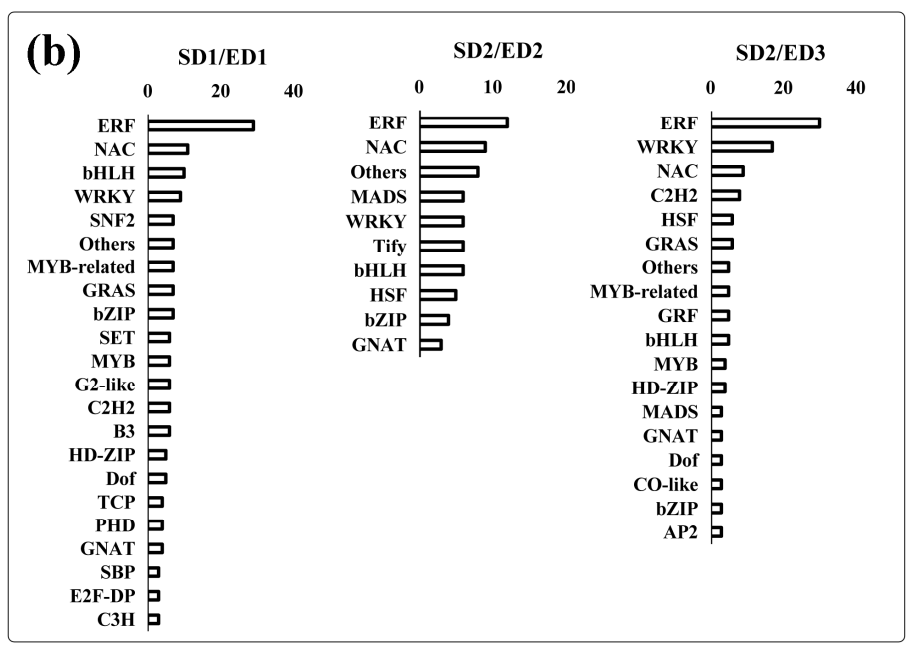
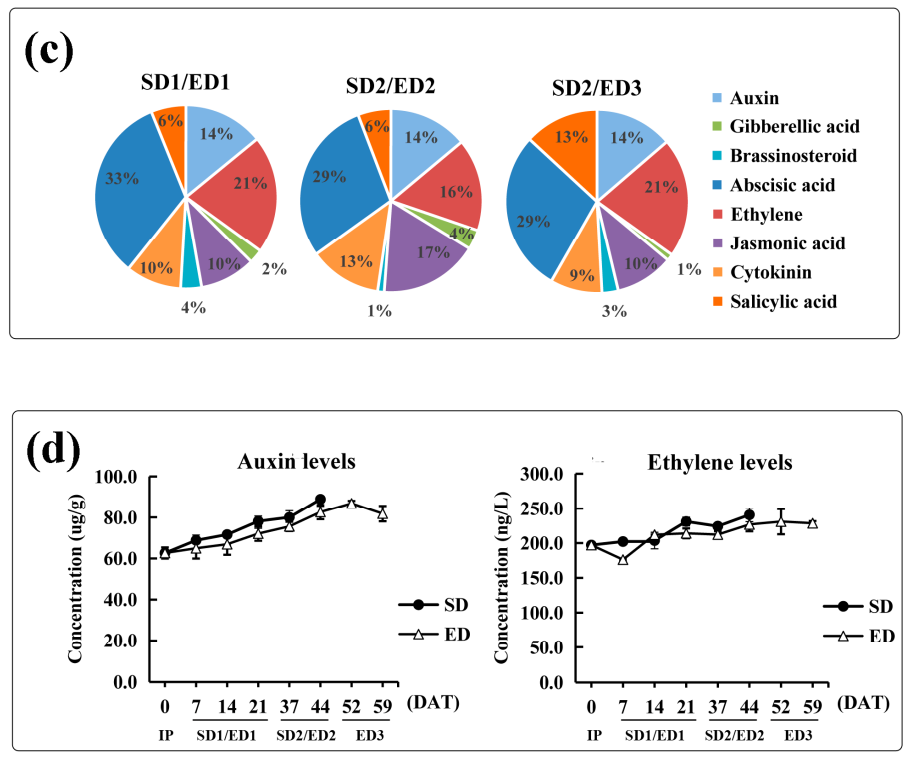

Figure 3. The overall analyses of DEGs in the three comparisons between SD and ED light cycles. (a) KEGG enrichment analysis of DEGs. All terms were listed ( $p$-value < 0.05). (b) The detailed information of transcription factors (TFs) in the DEGs. (c) The pie charts represent the percentage of DEGs (including up- and down-regulated DEGs) involved in phytohormone signal transduction entry. (d) The concentration of auxin and ethylene according to the sampling time of RNA-Seq.

\subsection{The SD Condition Accelerated the Accumulation of Carbohydrates}

The SD photoperiod regime can promote the early XIS cucumber flowering, and the specific mechanisms and genes need further analysis. We performed Venn analysis of the five comparison pairs (SD1/IP, SD2/IP, ED1/IP, ED2/IP and ED3/IP) using the significant DEGs ( $\mid \log _{2}$ Ratio $\mid \geq 1$ ). Finally, 251, 881, 196, 452 and 169 DEGs were only detected in $\mathrm{SD} 1 / \mathrm{IP}, \mathrm{SD} 2 / \mathrm{IP}, \mathrm{ED} 1 / \mathrm{IP}, \mathrm{ED} 2 / \mathrm{IP}$ and ED3/IP, among them including 192, 371, 72, 234 and 165 up-regulated ones respectively (Figure 4a). Noteworthy, more up-regulated DEGs were enriched in SD (563) than that in ED (471). Then the further analysis was carried out by the unique up-regulated DEGs. 

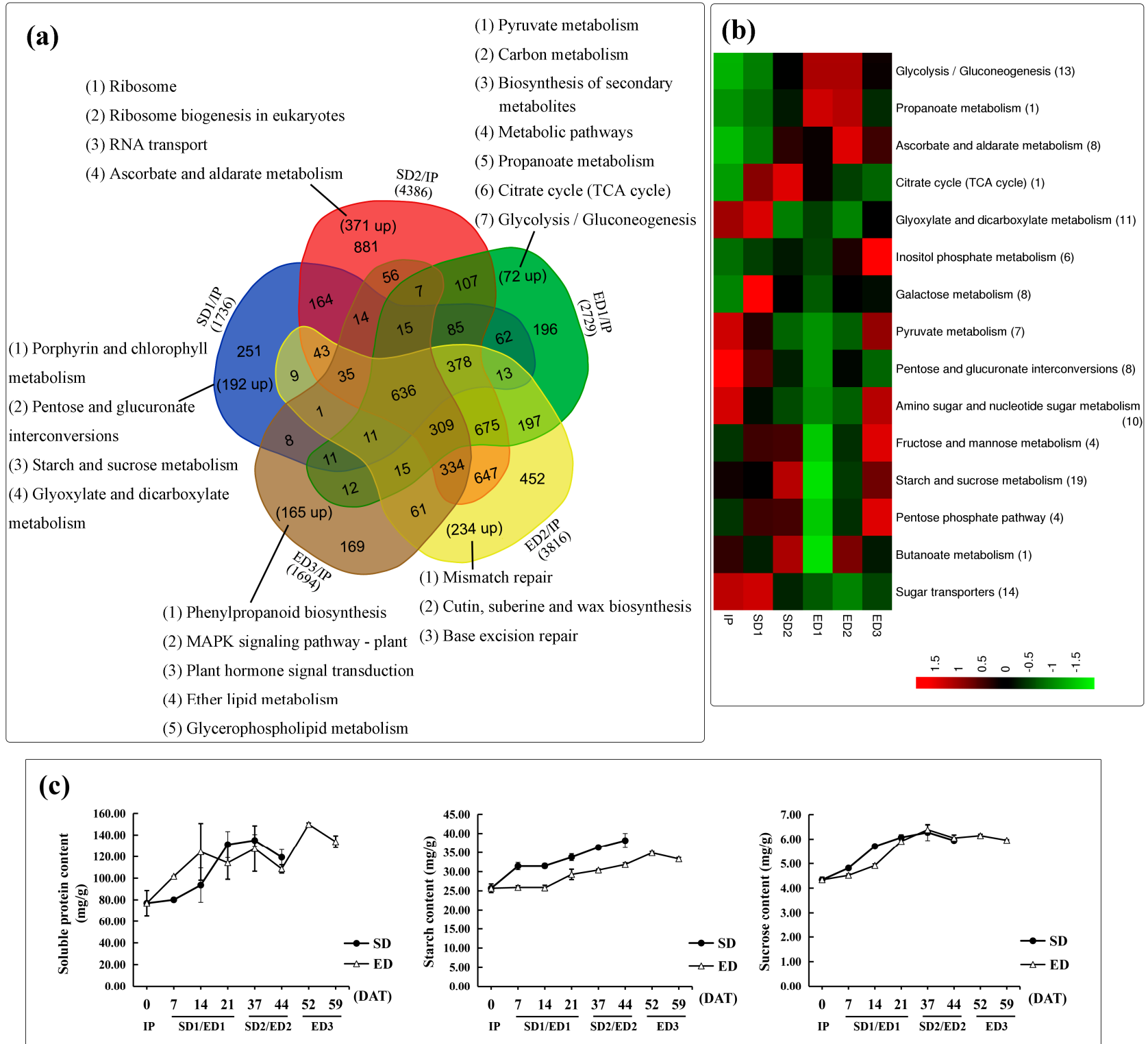

Figure 4. Identification of specific DEGs under SD and ED photoperiod treatments. (a) The Venn diagram showed the specific DEGs and its enrichment analysis (up-regulated genes) in the five comparisons (SD1/IP, SD2/IP, ED1/IP, ED2/IP and ED3/IP). (b) The expression patterns of carbohydrate-related DEGs in the six samples. (c) The content of soluble protein, starch and sucrose at the time points of RNA-Seq.

Although SD1 and ED1 were similar in their morphology of SAM (Figure 1c,f), DEGs were very different in SD1/IP and ED1/IP. Entries related to carbohydrates were specifically listed in SD1/IP (Pentose and glucuronate interconversions, Starch and sucrose metabolism and Glyoxylate and dicarboxylate metabolism) and ED1/IP (Pyruvate metabolism, Propanoate metabolism, Citrate cycle, Glycolysis/Gluconeogenesis), which provide nutrients for the flowering process (Figure 4a). The expression profile of carbohydrate-related DEGs were selected and presented, and finally in total of 114 DEGs were detected, including genes involved in carbohydrate biosynthesis, metabolic and transport (Figure 4b; Table S7). More DEGs began to hold a high expression level at SD1 under SD photoperiod regime; however, their high expression level were detected until ED3 stages under ED condition. Noteworthy, the number of DEGs in "Starch and sucrose metabolism" entry was the highest, so we detected the content of starch and sucrose. The measurement of starch and sucrose showed an increasing trend with the flowering process under both light cycles (Figure 4c). The sucrose content at SD1 was higher than that at ED1, 
which means the difference of sucrose appeared at the floral primordia initiation stage. The starch content under SD was always higher than that under ED, and was $22 \%$ higher at the highest point (14 DAT). The transport and storage of carbohydrates was delayed under ED, which was consistent with the delaying flowering under ED condition.

In SD2/IP, the KEGG enrichment analysis was obviously associated with translation process, corresponding to the higher content of soluble protein at SD2 (37 and 44 DAT) than that at ED2, including "ribosome", "ribosome biogenesis in eukaryotes" and "RNA transport" entries (Figure 4c). As the plant structural components, a variety of proteins need to be synthesized for the formation of floral organs. Ribosomes are the cellular factories responsible for making proteins [43]. The terms related to translation process and higher content of soluble protein at SD2 were consistent with the early development of floral organs under SD than ED regime.

3.6. Flowering Related Genes and Transcription Factors Showed Different Expression Patterns in Response to SD and ED Photoperiod Regimes

According to a previous study in the model plant Arabidopsis, homologs of floweringrelated genes were selected from the significant DEGs between SD and ED regimes, and their expression profiles were observed across six samples (Figure 5a; Table S8). DEGs related to multiple flowering pathways were detected, but no genes belong to the autonomous pathway. More DEGs (11) associated with the photoperiodic flowering network were detected, which further demonstrated that the photoperiodic pathway is the main flowering pathway of XIS cucumber SWCC8. It is worth noting that the flowering-promoting genes pseudo-response regulator 5 (PRR5) and LEAFY ( $L F Y)$ have higher expression levels especially under SD condition, which is consistent with the promoting flowering of SD photoperiod regime $[44,45]$.

(a)

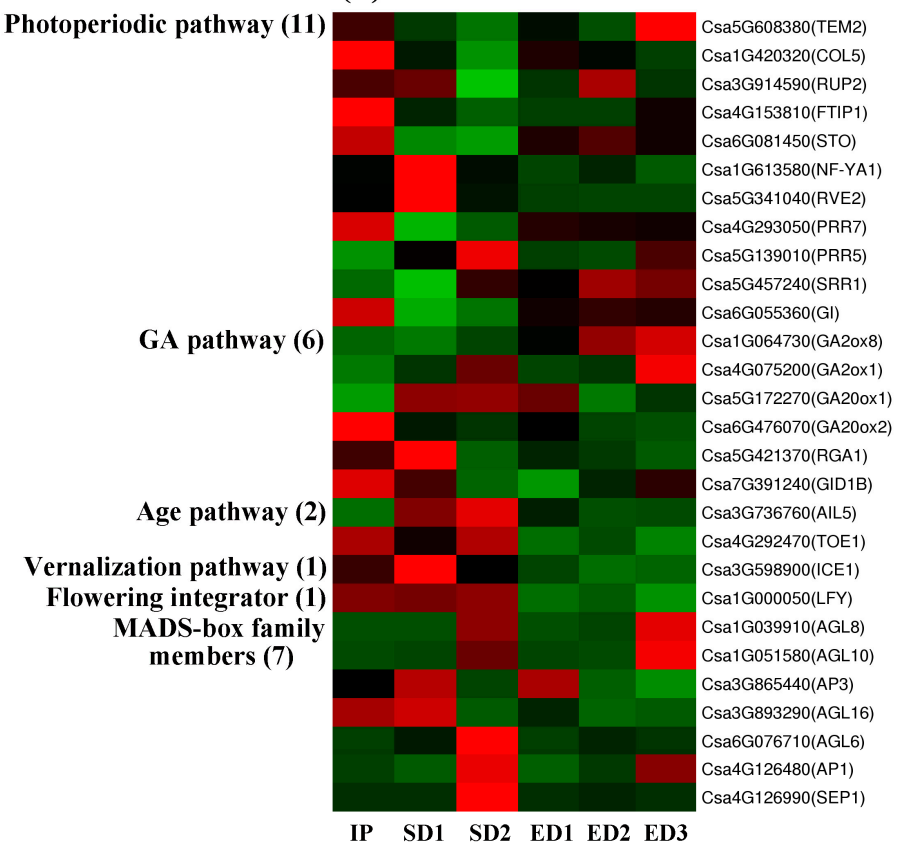

(b)

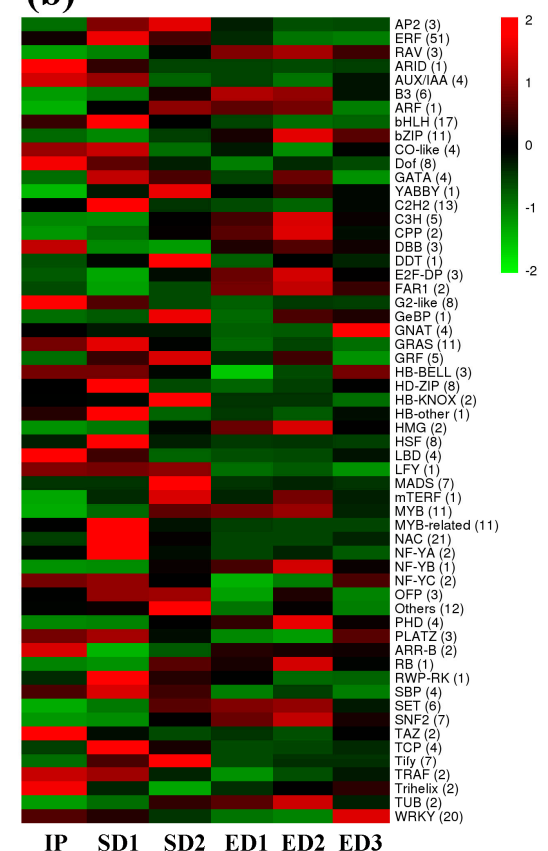

Figure 5. The expression profiles of DEGs related to the flowering pathways (a) and transcription factors (b).

The expression profiles of flowering-related TFs were compared between SD and ED conditions. Finally, 58 types and 338 TFs were detected in the significant DEGs (Figure 5b; Table S9). Because SD condition promotes the early flowering of XIS cucumber SWCC8, special attention was paid on the TFs which had high expression levels under SD regime. Among them, ERF (51), bHLH (17), $\mathrm{C}_{2} \mathrm{H}_{2}$ (13), CO-like (4), Dof (8), GATA (4), MYB-related (11), NAC (21) TFs had a higher expression level at SD1 stage; and AP2 (3), YABBY (1), 
GRF (8), MADS-box (7) TFs showed an upward regulation trend when they reached SD2 stage. As an important TFs in regulating the floral organ development [46], the expression patterns of MADS-box genes were listed in detail (Figure 5a). The expression level of MADS-box genes under SD was generally higher than that under ED, consistent with the early flowering under SD condition. For example, the expression levels of Csa4G126990 (SEP1) and Csa6G076710 (AGL6) were significantly higher than other genes especially at the SD2 stage. Additionally, three MADS-box transcription factors, Csa4G126480, Csa1G039910, Csa1G051580, exhibited an increase trend at the floral organ development stage (SD2 and ED3) under both light cycles, in agreement with the positive effects of MADS-box TFs in the flower development [46].

\subsection{A Co-Expression Network Was Constructed by CsaNFYA1 and Related Genes}

Analysis of the expression patterns of candidate gene CsaNFYA1 showed that its expression has been detected in multiple organs, and the expression level in reproductive organs, male flower, female flower and ovary, was relatively higher. The expression level of CsaNFYA1 was the highest in ovary and lowest in stem (Figure 6a). Transcriptome sequencing materials were used to determine the expression patterns of CsaNFYA1 under different photoperiod conditions. The results showed that the expression trend of CsaNFYA1 differed between SD and ED set-ups. The expression level of CsaNFYA1 didn't change much under ED condition. However, CsaNFYA1 had a significantly high expression level at SD1 stage, the floral primordia initiation stage, which suggests that CsaNFYA1 may promote floral transition under SD regime (Figure $6 \mathrm{~b}$ ).

(a)

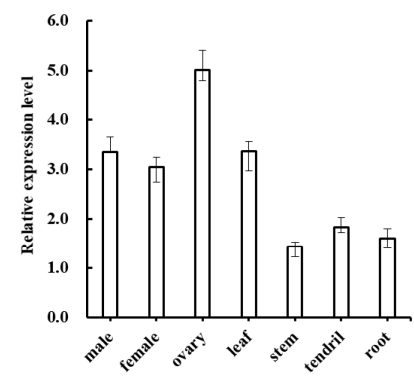

(b)

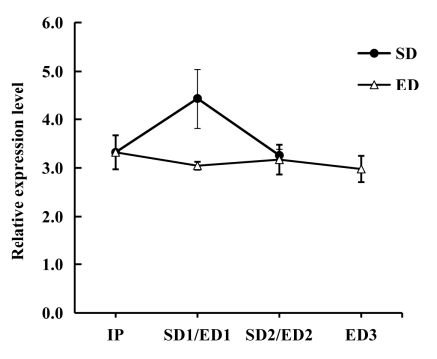

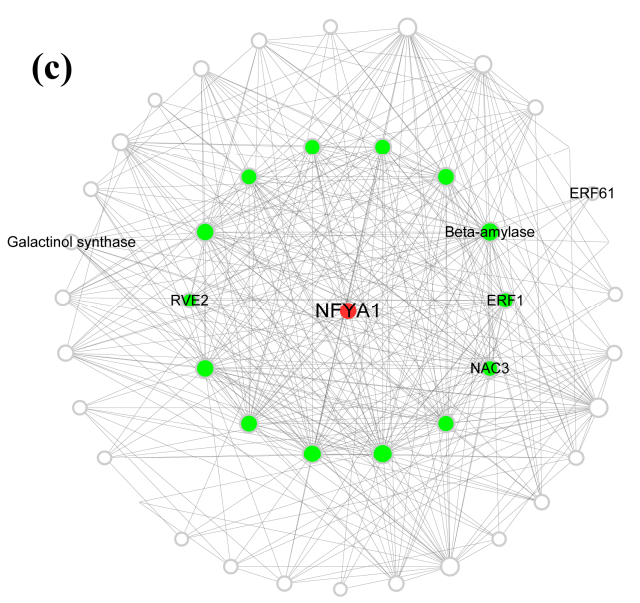

Figure 6. The verification of candidate gene CsaNFYA1. (a) The expression patterns of CsaNFYA1 in multiple organs. (b) The expression patterns of CsaNFYA1 under different photoperiod regimes. (c) The co-expression network of candidate gene CsaNFYA1 according to the WGCNA results. CsaNFYA1 was labeled by the red circle. Fourteen genes established the directed co-expression relationship with CsaNFYA1 were marked by green circles. Other genes in the network were in white circles. 
Using the WGCNA results (Table S10), we constructed a network to show the coexpression relationships of CsaNFYA1 and related genes, in which nodes represent genes and lines (edges) represent the coexpression relationships. CsaNFYA1 was identified in the 'mediumpurple 4' module, which has a high correlation with SD1 stage $(\mathrm{r}=0.93$, $p$-value $=1 \times 10^{-8}$; Figure S4). Finally, a total of 45 genes were used to construct the coexpression network and 14 (green circle) of them have a directed co-expression relationship with CsaNFYA1 (Figure 6c). Genes associated with multiple pathways were linked to CsaNFYA1, such as a $\beta$-amylase gene (Csa3G133950), which is associated with the starch catabolism. Noteworthy, the gene REVEILLE2 (RVE2, Csa5G341040), which regulates the photoperiod-mediated flowering time through the circadian clock pathway [47], also established a direct co-expression relationship with CsaNFYA1. In addition, the transcription factors ERF1 (Csa6G012810) and NAC3 (Csa3G101810) also had a direct co-expression relationship with CsaNFYA1.

\section{Discussion}

\subsection{QTLs on Flowering Time Are Conservative in Photoperiod-Sensitive Cucumbers}

Photoperiod sensitivity is a complex scientific problem, which is determined by numerous factors. A previous study on photoperiod sensitivity in maize identified 29 QTLs for plant height, leaf number, and flowering time. The three traits showed a strong correlation with each other, and they were mapped at a similar position on maize chromosomes [48]. In wheat, flowering and maturity time were mapped as a reference to measure photoperiod responses by two spring cultivars with completely different photoperiod sensitivity [49]. In addition, to reveal the genetic basis of photoperiod effect on short-day plant rice, the heading date (flowering time) was collected and analyzed [11,50]. Among them, time until flowering was used as a key indicator to study the photoperiod response [11,29]. In our study, we selected the days to first flowering to evaluate the effect of photoperiod in XIS cucumber.

Previous studies have reported the QTLs related to flowering time in cucumber (Table 5). For example, Sheng et al. [31] reported that FT1.1 and FT6.3 contribute to the flowering time by the cross of cucumber line Gy14 and wild cucumber line WI7221 (photoperiod sensitive). In addition, Pan et al. [30] and Bo et al. [17] identified three (FT1.1, FT5.1 and FT6.2) and two (fft1.1 and fft6.1) QTLs on flowering time, respectively by populations derived from across between semi-wild XIS cucumber (photoperiod-sensitive) and cultivated cucumber. According to the previous studies, it is worth noting that, when one of the parents is a cucumber variety that is sensitive to photoperiod, the mapping results on flowering time all contain the similar QTLs on chromosomes 1 and 6. That is, the QTLs on chromosomes 1 and 6 are two conservative QTLs related to the photoperiodmediated flowering time. In this study, the QTL-Seq analysis found four QTLs on the days to first flowering (dff1.1, dff3.1, dff6.1, dff7.1); but the SSR-based mapping only detected the QTLs on chromosome 1 (Sdff1.1 and Fdff1.1). Combined QTL-Seq, SSR-based mapping and previous studies together, it showed that there was a major-effect QTL of flowering time on chromosome 1, namely DFF1.1 in this study (Figure 2b).

\subsection{The Effect of Photoperiod on Flowering Process Is Different in Time}

Photoperiod has many effects on plant growth and development, but perhaps the most profound involves flowering [12,51], although which floral development stages are affected, floral primordia initiation, floral organ development (differentiation into sepal, petal, stamen, gynoecia) and anthesis are controversial. It has been reported that photoperiod affects the formation of floral primordia [52]. Huang et al. [53] observed that longer day-length promoted the floral transition in Arabidopsis. In opium poppy, photoperiod sensitivity continues from the initiation of flower buds to later floral organ development [54]. Previous genetic mapping work has focused on flowering time in relation to photoperiod response $[11,49,50]$. In the present study, both vegetative and reproductive growth were regulated by different photoperiods in XIS cucumber (Table 2; 
Figure 1). The major influence of photoperiod was most apparent in the later floral development and at the floral organ formation (Figure 1h-m), which in turn affects the days to first flowering. The reason for this could be that the effect of photoperiod on floral primordia occurs at a certain threshold. The range of photoperiod differences in this study, $8 \mathrm{~h} / 16 \mathrm{~h}$ and $12 \mathrm{~h} / 12 \mathrm{~h}$, had little effects on the initiation of floral primordia, but over time, the effects of photoperiod appeared to accumulate, evident as more pronounced effects in later development of floral organs.

\subsection{Various Types of Genes Are Involved in the Regulation of Photoperiodic Flowering}

Plant flowering is a complex process that is triggered by a combination of various internal and external cues [10]. The day-length signals stimulate the signal-receiving leaves, which stimulate the synthesis of sugars, hormones and other stimuli. Molecular genetic studies have uncovered multiple flowering-time loci and candidate genes, such as the florigen gene $F T$ [55], the zinc finger transcription factor $C O$ [8], a pseudo-response regulator protein DTH7 [11]. Additionally, El-Lithy et al. [56] confirmed the close relationship between carbohydrates and flowering in Arabidopsis using the genetic mapping. Krizek [40] and Yamaguchi et al. [41] reported the pivotal roles of auxin during the primordia initiation and development of flower. Previous study showed that the role of ethylene signal in the flowering process is mediated through the regulation of miR156 and its target gene $S P L$, and that ethylene response factors (ERFs) act as activators during the flowering process $[42,57,58]$.

NF-Y transcription factors, also known as heme-associated proteins (HAPs), are composed of three independent subunits: NF-YA (HAP2), NF-YB (HAP3), and NF-YC (HAP5) [59]. The general mechanism of NF-Y initiates with the histone-fold domain (HFD, including $N F-Y B$ and $N F-Y C$ ) and $N F-Y A$ which form a heterotrimeric complex; the complex then binds at the CCAAT motif of target gene $F T$ promoter activating its expression and initiating the flowering process [60-64]. NF-YA has the ability to identify the specific sequence of CCAAT-box in the FT promoter region [32]. Siriwardana et al. [33] proved that $N F-Y A$ mediated flowering positively in the photoperiod-dependent flowering pathway. However, Wenkel et al. [65] reported that the overexpression of NF-YA1/4 resulted in late flowering in Arabidopsis. In this study, CsaNFYA1 was treated as a candidate of the days to first flowering (Figure 2), and the expression profile showed that CsaNFYA1 plays positive roles in the flowering transition process (Figure $6 \mathrm{~b}$ ). Plant flowering is a complex regulatory process involving multiple flowering pathways and signaling molecules [66].

\section{Conclusions}

In this study, we detected a conservative QTL locus of DFF on chromosome 1. Combined QTL-Seq and re-sequencing data showed that CsaNFYA1 was considered as a potential candidate gene of DFF. Through the genetic and transcriptomic analysis, we found that genes specifically acting in flowering pathway, hormone and carbohydrate might cooperate with photoperiod signals to regulate XIS cucumber flowering. This paper sheds light on the specific genes that are affected by photoperiod sensitivity that affect plant flowering and provides useful information for the further study of flowering in photoperiod-sensitive plants.

Supplementary Materials: The following are available online at https: / www.mdpi.com/article / 10.3390/genes12071064/s1, Figure S1: Frequency distribution of the days to first flowering (DFF). Figure S2: Verify the expression profile of differential expressed genes (DEGs) in the RNA-Seq data by qRT-PCR. Figure S3: The correlation (a) and principal component analysis (b) of gene expression under SD and ED photoperiod regimes. Figure S4: The weighted gene co-expression network analysis (WGCNA) of genes in six samples of XIS cucumber. Table S1: Summary of primer sequences information. Table S2: Summary of QTL-Seq data for each sample. Table S3: Candidate genes predicted by QTL-Seq on chromosome 1. Table S4: The sequences analysis of 15 nonsynonymous mutation genes in the overlapping region (DFF1.1) by the resequencing data of nine cucumber varieties. Table S5: The introduction of flowering characteristics of nine cucumber varieties. Table S6: Summary of transcriptome sequencing data. Table S7: The expression patterns and annotation 
information of carbohydrate-related DEGs. Table S8: The expression patterns of flowering-related DEGs. Table S9: The expression levels of DEGs involved in transcription factors. Table S10: The expression level of genes participating into WGCNA.

Author Contributions: Conceptualization, J.C., J.L. and Z.T.; methodology, Z.T.; software, Z.T. and X.Q.; validation, Z.T. and F.Y.; formal analysis, Z.T.; investigation, Z.T. and F.Y.; data curation, Z.T. and J.L.; writing—original draft preparation, Z.T.; writing—review and editing, J.C., J.L., H.O.O. and M.J.; supervision, J.C., J.L. and M.J.; project administration, J.L.; funding acquisition, J.C. All authors have read and agreed to the published version of the manuscript.

Funding: This research was funded by the National Key Research and Development Program of China (Grant No. 2020YFE0202900), Asia Cooperation Fund Project: Green and efficient vegetable production demonstration park in Lancang-Mekon river region-2021, Jiangsu Belt and Road innovation cooperation project: Cooperative development of overseas application of molecular breeding technology in horticultural crops (BZ2019012). A Project Funded by the Priority Academic Program Development of Jiangsu Higher Education Institutions.

Institutional Review Board Statement: Not applicable.

Informed Consent Statement: Not applicable.

Data Availability Statement: Not applicable.

Acknowledgments: We thank Zhai, Y.F., Wang, X. and Wang, P.Q. for their help in bioinformatics related analysis.

Conflicts of Interest: The authors declare no conflict of interest.

\section{References}

1. Amasino, R.M.; Michaels, S.D. The timing of flowering. Plant Physiol. 2010, 154, 516-520. [CrossRef]

2. Song, Y.H.; Shim, J.S.; Kinmonth-Schultz, H.A.; Imaizumi, T. Photoperiodic flowering: Time measurement mechanisms in leaves. Annu. Rev. Plant Biol. 2015, 66, 441-464. [CrossRef]

3. Koornneef, M.; Alonso-Blanco, C.; Peeters, A.J.M.; Soppe, W. Genetic control of flowering time in Arabidopsis. Annu. Rev. Plant Physiol. Plant Mol. Biol. 1998, 49, 345-370. [CrossRef] [PubMed]

4. Hayama, R.; Yokoi, S.; Tamaki, S.; Yano, M.; Shimamoto, K. Adaptation of photoperiodic control pathways produces short-day flowering in rice. Nature 2003, 422, 719-722. [CrossRef]

5. Weller, J.L.; Vander Schoor, J.K.; Perez-Wright, E.C.; Hecht, V.; Gonzalez, A.M.; Capel, C.; Yuste-Lisbona, F.J.; Lozano, R.; Santalla, M. Parallel origins of photoperiod adaptation following dual domestications of common bean. J. Exp. Bot. 2019, 70, 1209-1219. [CrossRef] [PubMed]

6. Corbesier, L.; Vincent, C.; Jang, S.H.; Fornara, F.; Fan, Q.Z.; Searle, I.; Giakountis, A.; Farrona, S.; Gissot, L.; Turnbull, C.; et al. FT protein movement contributes to long-distance signaling in floral induction of Arabidopsis. Science 2007, 316, 1030-1033. [CrossRef] [PubMed]

7. Tamaki, S.; Matsuo, S.; Wong, H.L.; Yokoi, S.; Shimamoto, K. Hd3a protein is a mobile flowering signal in rice. Science 2007, 316, 1033-1036. [CrossRef] [PubMed]

8. Putterill, J.; Robson, F.; Lee, K.; Simon, R.; Coupland, G. The CONSTANS gene of Arabidopsis promotes flowering and encodes a protein showing similarities to zinc finger transcription factors. Cell 1995, 80, 847-857. [CrossRef]

9. Zhao, H.; Wu, D.; Kong, F.Y.; Lin, K.; Zhang, H.S.; Li, G. The Arabidopsis thaliana Nuclear Factor Y transcription factors. Front. Plant Sci. 2017, 7, 2045. [CrossRef]

10. Srikanth, A.; Schmid, M. Regulation of flowering time: All roads lead to Rome. Cell Mol. Life Sci. 2011, 68, 2013-2037. [CrossRef]

11. Gao, H.; Jin, M.N.; Zheng, X.M.; Chen, J.; Yuan, D.Y.; Xin, Y.Y.; Wang, M.Q.; Huang, D.Y.; Zhang, Z.; Zhou, K.N.; et al. Days to heading 7, a major quantitative locus determining photoperiod sensitivity and regional adaptation in rice. Proc. Natl. Acad. Sci. USA 2014, 111, 16337-16342. [CrossRef]

12. Alvarez-Buylla, E.R.; Benitez, M.; Corvera-Poire, A.; Chaos Cador, A.; de Folter, S.; Gamboa de Buen, A.; Garay-Arroyo, A.; Garcia-Ponce, B.; Jaimes-Miranda, F.; Perez-Ruiz, R.V.; et al. Flower development. Arab. Book 2010, 8, e0127. [CrossRef]

13. Qi, C.Z.; Yuan, Z.Z.; Li, Y.X. A new type of cucumber-Xishuangbanna cucumber. Acta Hortic. Sin. 1983, 10, $259-264$.

14. Chen, J.F.; Zhang, S.L.; Zhang, X.G. The Xishuangbanna gourd (Cucumis sativus L. var. xishuangbannanesis Qi et Yuan), a traditionally cultivated plant of the Hanai people, Xishuangbanna, Yunnan, China. Cucurbit Genet. Coop. Rep. 1994, 17, 18-20.

15. Shen, D.; Li, X.X.; Song, J.P.; Wang, H.P.; Qiu, Y. Effects of different sowing dates on sex type and lateral stem development of Xishuangbanna cucumber. China Veget. 2011, 6, 22-27.

16. Bo, K.L.; Chen, L.Z.; Qian, C.T.; Zhang, S.X.; Chen, J.F. Short-day treatments induce flowering of Xishuangbanna cucumber. China Cucurbits Veg. 2010, 23, 1-3. 
17. Bo, K.L.; Ma, Z.; Chen, J.F.; Weng, Y.Q. Molecular mapping reveals structural rearrangements and quantitative trait loci underlying traits with local adaptation in semi-wild Xishuangbanna cucumber (Cucumis sativus L. var. xishuangbannanesis Qi et Yuan). Theor. Appl. Genet. 2015, 128, 25-39. [CrossRef] [PubMed]

18. Zeng, Z.B. Precision mapping of quantitative trait loci. Genetics 1994, 136, 1457-1468. [CrossRef] [PubMed]

19. Silva, L.D.C.E.; Wang, S.C.; Zeng, Z.B. Composite interval mapping and multiple interval mapping: Procedures and guidelines for using Windows QTL Cartographer. Methods Mol. Biol. 2012, 871, 75-119.

20. Murray, M.G.; Thompson, W.F. Rapid isolation of high molecular weight plant DNA. Nucleic Acids Res. 1980, 8, 4321-4325. [CrossRef]

21. Huang, S.; Li, R.; Zhang, Z.; Li, L.I.; Gu, X.; Fan, W.; Lucas, W.J.; Wang, X.; Xie, B.; Ni, P.; et al. The genome of the cucumber, Cucumis sativus L. Nat. Genet. 2009, 41, 1275-1281. [CrossRef] [PubMed]

22. Malatesta, M. Histological and histochemical methods-Theory and practice. Eur. J. Histochem. 2016, 60. [CrossRef]

23. Trapnell, C.; Pachter, L.; Salzberg, S.L. TopHat: Discovering splice junctions with RNA-Seq. Bioinformatics 2009, $25,1105-1111$. [CrossRef] [PubMed]

24. Mortazavi, A.; Williams, B.A.; McCue, K.; Schaeffer, L.; Wold, B. Mapping and quantifying mammalian transcriptomes by RNA-Seq. Nat. Methods 2008, 5, 621-628. [CrossRef]

25. Wang, L.K.; Feng, Z.X.; Wang, X.; Wang, X.W.; Zhang, X.G. DEGseq: An R package for identifying differentially expressed genes from RNA-seq data. Bioinformatics 2010, 26, 136-138. [CrossRef] [PubMed]

26. Livak, K.J.; Schmittgen, T.D. Analysis of relative gene expression data using real-time quantitative PCR and the $2^{-\Delta \Delta C T}$ method. Methods 2001, 25, 402-408. [CrossRef]

27. Bradford, M.M. A rapid and sensitive method for the quantitation of microgram quantities of protein utilizing the principle of protein-dye binding. Anal. Biochem. 1976, 72, 248-254. [CrossRef]

28. Rosa, M.; Hilal, M.; Gonzalez, J.A.; Prado, F.E. Low-temperature effect on enzyme activities involved in sucrose-starch partitioning in salt-stressed and salt-acclimated cotyledons of quinoa (Chenopodium quinoa Willd.) seedlings. Plant Physiol. Biochem. 2009, 47, 300-307. [CrossRef]

29. Yano, M.; Katayose, Y.; Ashikari, M.; Yamanouchi, U.; Monna, L.; Fuse, T.; Baba, T.; Yamamoto, K.; Umehara, Y.; Nagamura, Y.; et al. $H d 1$, a major photoperiod sensitivity quantitative trait locus in rice, is closely related to the Arabidopsis flowering time gene CONSTANS. Plant Cell 2000, 12, 2473-2483. [CrossRef]

30. Pan, Y.P.; Qu, S.P.; Bo, K.L.; Gao, M.L.; Haider, K.R.; Weng, Y.Q. QTL mapping of domestication and diversifying selection related traits in round-fruited semi-wild Xishuangbanna cucumber (Cucumis sativus L. var. xishuangbannanesis). Theor. Appl. Genet. 2017, 130, 1531-1548. [CrossRef]

31. Sheng, Y.Y.; Pan, Y.P.; Li, Y.H.; Yang, L.M.; Weng, Y.Q. Quantitative trait loci for fruit size and flowering time-related traits under domestication and diversifying selection in cucumber (Cucumis sativus). Plant Breed. 2020, 139, 176-191. [CrossRef]

32. Nardini, M.; Gnesutta, N.; Donati, G.; Gatta, R.; Forni, C.; Fossati, A.; Vonrhein, C.; Moras, D.; Romier, C.; Bolognesi, M.; et al. Sequence-specific transcription factor NF-Y displays histone-like DNA binding and H2B-like ubiquitination. Cell 2013, 152, 132-143. [CrossRef]

33. Siriwardana, C.L.; Gnesutta, N.; Kumimoto, R.W.; Jones, D.S.; Myers, Z.A.; Mantovani, R.; Holt, B.F. NUCLEAR FACTOR Y, subunit A (NF-YA) proteins positively regulate flowering and act through FLOWERING LOCUS T. PLoS Genet. 2016, 12, e1006496. [CrossRef] [PubMed]

34. Chen, K.; Rajewsky, N. The evolution of gene regulation by transcription factors and microRNAs. Nat. Rev. Genet. 2007, 8, 93-103. [CrossRef]

35. Ren, L.P.; Liu, T.; Cheng, Y.; Sun, J.; Gao, J.J.; Dong, B.; Chen, S.M.; Chen, F.D.; Jiang, J.F. Transcriptomic analysis of differentially expressed genes in the floral transition of the summer flowering chrysanthemum. BMC Genom. 2016, 17, 673. [CrossRef]

36. Wang, H.P.; Li, Y.; Pan, J.J.; Lou, D.J.; Hu, Y.R.; Yu, D.Q. The bHLH transcription factors MYC2, MYC3, and MYC4 are required for jasmonate-mediated inhibition of flowering in Arabidopsis. Mol. Plant 2017, 10, 1461-1464. [CrossRef]

37. Zhu, L.; Guan, Y.X.; Liu, Y.N.; Zhang, Z.H.; Jaffar, M.A.; Song, A.P.; Chen, S.M.; Jiang, J.F.; Chen, F.D. Regulation of flowering time in chrysanthemum by the R2R3 MYB transcription factor CmMYB2 is associated with changes in gibberellin metabolism. Hortic. Res. 2020, 7, 96. [CrossRef] [PubMed]

38. Krizek, B.A. AINTEGUMENTA and AINTEGUMENTA-LIKE6 act redundantly to regulate Arabidopsis floral growth and patterning. Plant Physiol. 2009, 150, 1916-1929. [CrossRef] [PubMed]

39. Dai, F.W.; Zhang, C.Q.; Jiang, X.Q.; Kang, M.; Yin, X.; Lu, P.T.; Zhang, X.; Zheng, Y.; Gao, J.P. RhNAC2 and RhEXPA4 are involved in the regulation of dehydration tolerance during the expansion of rose petals. Plant Physiol. 2012, 160, 2064-2082. [CrossRef]

40. Krizek, B.A. Auxin regulation of Arabidopsis flower development involves members of the AINTEGUMENTA-LIKE/PLETHORA (AIL/PLT) family. J. Exp. Bot. 2011, 62, 3311-3319. [CrossRef]

41. Yamaguchi, N.; Wu, M.F.; Winter, C.M.; Berns, M.C.; Nole-Wilson, S.; Yamaguchi, A.; Coupland, G.; Krizek, B.A.; Wagner, D. A molecular framework for auxin-mediated initiation of flower primordia. Dev. Cell 2013, 24, 271-282. [CrossRef] [PubMed]

42. Iqbal, N.; Khan, N.A.; Ferrante, A.; Trivellini, A.; Francini, A.; Khan, M.I.R. Ethylene role in plant growth, development and senescence: Interaction with other phytohormones. Front. Plant Sci. 2017, 8, 475. [CrossRef] [PubMed]

43. Wilson, D.N.; Cate, J.H.D. The structure and function of the eukaryotic ribosome. Cold Spring Harb. Perspect. Biol. 2012, 4, a011536. [CrossRef] [PubMed] 
44. Nakamichi, N.; Kita, M.; Niinuma, K.; Ito, S.; Yamashino, T.; Mizoguchi, T.; Mizuno, T. Arabidopsis clock-associated pseudoresponse regulators $P R R 9, P R R 7$ and PRR5 coordinately and positively regulate flowering time through the canonical CONSTANSdependent photoperiodic pathway. Plant Cell Physiol. 2007, 48, 822-832. [CrossRef]

45. Winter, C.M.; Austin, R.S.; Blanvillain-Baufume, S.; Reback, M.A.; Monniaux, M.; Wu, M.F.; Sang, Y.; Yamaguchi, A.; Yamaguchi, N.; Parker, J.E.; et al. LEAFY target genes reveal floral regulatory logic, cis motifs, and a link to biotic stimulus response. Dev. Cell 2011, 20, 430-443. [CrossRef]

46. Smaczniak, C.; Immink, R.G.H.; Muino, J.M.; Blanvillain, R.; Busscher, M.; Busscher-Lange, J.; Dinh, Q.D.; Liu, S.J.; Westphal, A.H.; Boeren, S.; et al. Characterization of MADS-domain transcription factor complexes in Arabidopsis flower development. Proc. Natl. Acad. Sci. USA 2012, 109, 1560-1565. [CrossRef]

47. Zhang, X.B.; Chen, Y.H.; Wang, Z.Y.; Chen, Z.L.; Gu, H.Y.; Qu, L.J. Constitutive expression of CIR1 (RVE2) affects several circadian-regulated processes and seed germination in Arabidopsis. Plant J. 2007, 51, 512-525. [CrossRef] [PubMed]

48. Wang, C.L.; Cheng, F.F.; Sun, Z.H.; Tang, J.H.; Wu, L.C.; Ku, L.X.; Chen, Y.H. Genetic analysis of photoperiod sensitivity in a tropical by temperate maize recombinant inbred population using molecular markers. Theor. Appl. Genet. 2008, 117, 1129-1139. [CrossRef] [PubMed]

49. Kamran, A.; Iqbal, M.; Navabi, A.; Randhawa, H.; Pozniak, C.; Spaner, D. Earliness per se QTLs and their interaction with the photoperiod insensitive allele Ppd-D1a in the Cutler x AC Barrie spring wheat population. Theor. Appl. Genet. 2013, 126, 1965-1976. [CrossRef]

50. Matsubara, K.; Kono, I.; Hori, K.; Nonoue, Y.; Ono, N.; Shomura, A.; Mizubayashi, T.; Yamamoto, S.; Yamanouchi, U.; Shirasawa, K.; et al. Novel QTLs for photoperiodic flowering revealed by using reciprocal backcross inbred lines from crosses between japonica rice cultivars. Theor. Appl. Genet. 2008, 117, 935-945. [CrossRef]

51. Thomas, B.; Vince-Prue, D. Photoperiodic Control of Flower Initiation. In Photoperiodism in Plants, 2nd ed.; Academic Press: London, UK, 1997; pp. 3-257.

52. Jeong, S.; Clark, S.E. Photoperiod regulates flower meristem development in Arabidopsis thaliana. Genetics 2005, 169, 907-915. [CrossRef]

53. Huang, F.; Yuan, W.Y.; Tian, S.; Zheng, Q.J.; He, Y.H. SIN3 LIKE genes mediate long-day induction of flowering but inhibit the floral transition in short days through histone deacetylation in Arabidopsis. Plant J. 2019, 100, 101-113. [CrossRef] [PubMed]

54. Wang, Z.C.; Acock, M.C.; Acock, B. Photoperiod sensitivity during flower development of opium poppy (Papaver somniferum L.). Ann. Bot. 1997, 79, 129-132. [CrossRef]

55. Wang, S.H.; Li, H.B.; Li, Y.Y.; Li, Z.; Qi, J.J.; Lin, T.; Yang, X.Y.; Zhang, Z.H.; Huang, S.W. FLOWERING LOCUS T improves cucumber adaptation to higher latitudes. Plant Physiol. 2020, 182, 908-918. [CrossRef] [PubMed]

56. El-Lithy, M.E.; Reymond, M.; Stich, B.; Koornneef, M.; Vreugdenhil, D. Relation among plant growth, carbohydrates and flowering time in the Arabidopsis Landsberg erecta x Kondara recombinant inbred line population. Plant Cell Environ. 2010, 33, 1369-1382. [CrossRef]

57. Fornara, F.; Coupland, G. Plant phase transitions make a SPLash. Cell 2009, 138, 625-627. [CrossRef] [PubMed]

58. Schaller, G.E. Ethylene and the regulation of plant development. BMC Biol. 2012, 10, 9. [CrossRef]

59. Mantovani, R. The molecular biology of the CCAAT-binding factor NF-Y. Gene 1999, 239, 15-27. [CrossRef]

60. Sinha, S.; Maity, S.N.; Lu, J.; de Crombrugghe, B. Recombinant rat CBF-C, the third subunit of CBF/NFY, allows formation of a protein-DNA complex with CBF-A and CBF-B and with yeast HAP2 and HAP3. Proc. Natl. Acad. Sci. USA 1995, 92, 1624-1628. [CrossRef]

61. Sinha, S.; Kim, I.S.; Sohn, K.Y.; de Crombrugghe, B.; Maity, S.N. Three classes of mutations in the A subunit of the CCAAT-binding factor CBF delineate functional domains involved in the three-step assembly of the CBF-DNA complex. Mol. Cell Biol. 1996, 16, 328-337. [CrossRef]

62. Ben-Naim, O.; Eshed, R.; Parnis, A.; Teper-Bamnolker, P.; Shalit, A.; Coupland, G.; Samach, A.; Lifschitz, E. The CCAAT binding factor can mediate interactions between CONSTANS-like proteins and DNA. Plant J. 2006, 46, 462-476. [CrossRef] [PubMed]

63. Kumimoto, R.W.; Adam, L.; Hymus, G.J.; Repetti, P.P.; Reuber, T.L.; Marion, C.M.; Hempel, F.D.; Ratcliffe, O.J. The Nuclear Factor Y subunits NF-YB2 and NF-YB3 play additive roles in the promotion of flowering by inductive long-day photoperiods in Arabidopsis. Planta 2008, 228, 709-723. [CrossRef] [PubMed]

64. Cao, S.; Kumimoto, R.W.; Gnesutta, N.; Calogero, A.M.; Mantovani, R.; Holt, B.F. A distal CCAAT/NUCLEAR FACTOR Y complex promotes chromatin looping at the FLOWERING LOCUS T promoter and regulates the timing of flowering in Arabidopsis. Plant Cell 2014, 26, 1009-1017. [CrossRef] [PubMed]

65. Wenkel, S.; Turck, F.; Singer, K.; Gissot, L.; Le Gourrierec, J.; Samach, A.; Coupland, G. CONSTANS and the CCAAT box binding complex share a functionally important domain and interact to regulate flowering of Arabidopsis. Plant Cell 2006, 18, 2971-2984. [CrossRef]

66. Xing, L.B.; Zhang, D.; Li, Y.M.; Shen, Y.W.; Zhao, C.P.; Ma, J.J.; An, N.; Han, M.Y. Transcription profiles reveal sugar and hormone signaling pathways mediating flower induction in Apple (Malus domestica Borkh.). Plant Cell Physiol. 2015, 56, $2052-2068$. [CrossRef] [PubMed] 